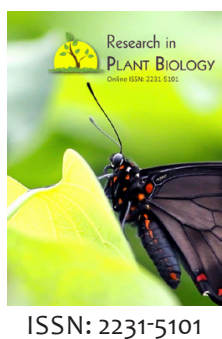

Received: February 03, 2019 Accepted: March 18, 2019 Published: March 24, 2019

\author{
*Corresponding Author: \\ Chaitali Roy \\ Email: chaitalli.bi@jcbose.ac.in
}

\section{Stress causing dynamic changes of four phytohormones in tobacco and tomato: A GC-MS analysis}

\author{
Chaitali Roy ${ }^{1 *}$, Smriti Ranjan Maji², Tapas Kumar Ghose', \\ Swati Gupta Bhattacharya'
}

'Division of Plant Biology, Bose Institute, 93/1 Acharya Prafulla Chandra Road, Kolkata 700009, India, ${ }^{2} \mathrm{CIF}, \mathrm{P}-1 / 12$, C.I.T. Scheme VII M, Bose Institute, Kolkata 700054, India

\begin{abstract}
Many analytical methods are in use to analyse plant hormones in different plants. Here this work provides a sensitive, accurate and quite accessible GC-MS (gas chromatography/mass spectrometry) method to quantify phytohormones indole-3-aceticacid (IAA), abscisic acid (ABA), jasmonic acid (JA) and salicylic acid (SA). These signaling molecules were analysed in two different plants, tomato and tobacco grown in vitro. The protocol designed to assess, how abiotic stress brings about changes in the level of endogenous hormones in the leaves of both the plants under study. A hormone profile of salt stressed leaves shows that different plant hormones are involved in diverse physiologicsl processes. Crosstalk between these hormones result in synergetic or antagonic interactions which have important roles to play in abiotic stress response.
\end{abstract}

KEYWORDS: Phytohormones, Abscisic acid, Indole-3-acetic acid, Jasmonic acid, Salicylic acid

\section{INTRODUCTION}

The Solanaceae include tobacco (Nicotiana tabacum L.) and tomato (Solanum lycopersicum L.). Tobacco and tomato are agriculturally important Solanaceae crops which are extensively studied [1-3]. Being sessile, plants as defense mechanism can rapidly modify in response to the changes in their surroudings such as drought, flood, salinity, shading or low/high temperature. Such stresses often found to be the major causes to affect plant production. Salinity stress is one of such leading causes for crop losses in the field. In order to give impetus to the stress-responsemechanism in tobacco and tomato, it is essential to understand their response to different abiotic stresses at multiple levels.

Flexibility in the growth patterns of plants is partly achieved by the action of phytohormones. They together form a signaling network which regulates plant response to different abiotic as well as biotic stresses. Several reviews in this regard add to the recent knowledge of hormonal cross-talk responsible for plant stress responses [4-6]. Plant hormones are the signaling chemicals which control almost all aspects of plant life.

Plants have successfully evolved through their developmental processes to face the challenges of environmental cues. Several hormones play major roles in abiotic (Figure 1) and biotic stress responses, where ABA is the key player. This hormone has a major role in stress signaling causing an immediate response like hydraulic signal that triggers ABA biosynthesis in the system [7]. Phytohormones can mediate a wide range of plants' responses, from rapid (e.g. stomata closure) to long term adaptations by modulating the programs of plant growth and development. Cytokinins and auxins are predominantly positive regulators of cell division and growth. Abscisic acid as a growth inhibitor, acts in stress conditions like drought.

Plant hormones have an important role in the response mechanism against abiotic stress [8]. Stress ultimately may result into retarded growth thus the plant can focus its resources on combating the stress [9]. In nature, plants fight against stresses by modulating various physiological, biochemical and molecular actions. These counter-actions lead to alterations in gene expression, regulation of biogenesis, changes of cellular metabolite levels and changes in ion homeostasis. Regulation of gene at the transcription level is one of the major control points in biological system. These growth-regulators and transcription-factors are the key players in this process [10]. ABA as a stress-responsive signaling molecule is the most well studied hormone in the past decade. In the recent past, a lot of research has been done in the field of elucidation of the core $\mathrm{ABA}$ signaling pathway and proper identification of ABA receptors.

Copyright: () Roy, et al. This article is open access and licensed under the terms of the Creative Commons Attribution License (http://creativecommons.org/licenses/by/4.0/) which permits unrestricted, use, distribution and reproduction in any medium, or format for any purpose, even commercially provided the work is properly cited. Attribution - You must give appropriate credit, provide a link to the license, and indicate if changes were made. 


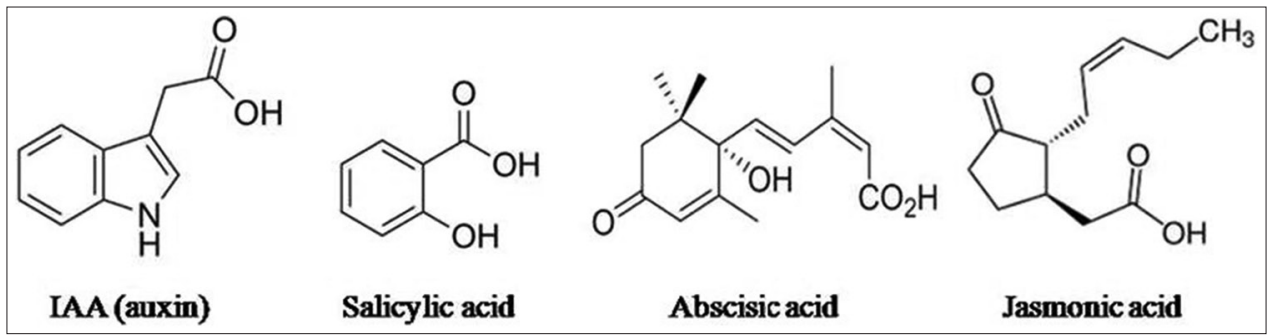

Figure 1: Chemical structures of important plant tohormones involved in abiotic stress response and tolerance

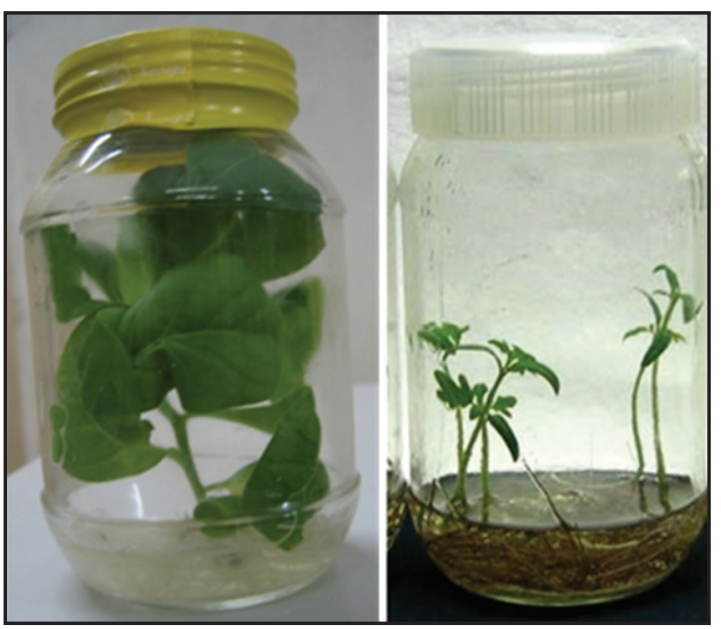

Figure 2: Tobacco and tomato plants in laboratory condition

For example, less water availability is first confronted by the plant roots which results in stomatal closure of leaf and thereby resulting into reduced transpiration to a great extent by the action of the stress hormone ABA [11]. Many recent experiments on plant hormones have shown that some hormones (such as ABA, auxins, cytokinins, SA, JAs, brassinosteroids etc.) have the potential to elevate the abiotic stress tolerance in various plant species [12]. Such hormonal responses are fundamental to the plant growth and development. In addition to their regulatory functions in development they have also key roles to play in coordinating different signal transduction pathways in environmental stresss responses [13].

The analysis of plants/mutants with an altered phytohormone profile has uncovered a high degree of interactions between auxins and cytokinins, abscisic acid and ethylene [14], jasmonates and ethylene [15], brassinolides and jasmonates [16], auxins and ethylene [17, 18], ethylene and cytokinin [19] or gibberellins and auxin [20].

Generally, the use of mass spectrometry (MS) is often coupled with an appropriate separation method like HPLC or GC $[21,22]$. Both the techniques are powerful enough to detect trace amounts of organic compounds even at the level of picogram $\left(10^{-12 \mathrm{~g}}\right)$ or femtogram $\left(10^{-15} \mathrm{~g}\right)$.

This study describes a simple method with extensive potential applicability. An objective of GC-MS based analysis of biochemical signals is to uncover the complex interaction and intensive crosstalk between ABA, IAA, SA and JA during salinity stress that can modulate the levels of these growth factors in the aerial parts of plants.

\section{MATERIALS AND METHODS}

\section{Plants and Growth Condition}

Two plant species (Figure 2) were used for the experiment. Tobacco (Nicotiana tabacum) cv SR1, Tomato (Solanum lycopersicum) cv Punjab Keshari. Tomato, and tobacco seeds were surface sterilized with $0.1 \%(\mathrm{w} / \mathrm{v}) \mathrm{HgCl}_{2}$ for 10 minutes then rinsed thoroughly and imbibed in sterile water for 6 to 8 hours and finally spread over sterile wet blotting papers in petriplates. Plates were kept in dark at $26^{\circ} \mathrm{C}$. Seeds were germinated in aseptic condition in the tissue culture room within 3 to 7 days. Seedlings were transferred in $0.25 \mathrm{X}$ Murashige \& Skoog [23] liquid medium (Hi-media) and grown for another 15 days $\left(16 \mathrm{~h}\right.$ dark and $8 \mathrm{~h}$ light period, $25-26^{\circ} \mathrm{C}$ ). The 15 day old plantlets were cultured in $0.5 \mathrm{X}$ Murashige \& Skoog liquid medium with or without salt $(150 \mathrm{mM} \mathrm{NaCl}$ solution) and kept on observation for a month. The media were replaced weekly.

\section{Harvest and Extraction of Plant Material}

We evaluated four growth factors in terms of reproducibility, carryover and linearity. Our attempt was to develop a simple but near accurate method as much as possible for the detection of selected non-polar compounds in leaf tissues of two different plants. To achieve the goal, we adopted the extraction procedure routinely used in our lab (Muller's method with modification) [21] for GC-MS analysis which has been found reliable method for the simultaneous monitoring of different plant hormones during physiological processes.

For the experiment, 200mg of leaf tissue were harvested from 30 days' culture of two plant species in Murashige and Skoog medium. Healthy leaf tissues of plants were cut off with a scissor which were found of typical stature and absolutely free from any signs of senescence and were immediately weighed on a Sartorius weighing tool. The desired parts immediately were ground in porcelain mortar-pestle using solvent (hot methanol). After that the homogenized tissue was incubated in the solvent for 1 hour at room temperature and the resulting fluid centrifuged at 10,000 rpm to remove cell debris. After centrifugation, the debris-free supernatant was decanted and transferred into an eppendorf tube. $1 \mathrm{ml}$ of ethylacetate was 
added in each tube. All samples were analysed immediately to prevent any degradation of facile phytohormones.

All samples were performed in triplicate. We always determine the optimum level of standard by a preliminary analysis of representative samples in a first run before the actual experiment for quantitation. Four standard solutions were prepared.

Stock solutions as standard of original phytohormones were prepared at $1 \mathrm{mg} / \mathrm{ml}$ in methanol. For calibration and comparison, working solutions of standards were prepared diluting stock solutions in methanol: water (7:3), at different concentration for each phytohormone depending on the range of the calibration curve.

IAA and ABA $(100 \mu \mathrm{g} / \mathrm{ml})$, SA and JA $(200 \mu \mathrm{g} / \mathrm{ml})$.

\section{Gas chromatography - mass spectrometry}

The compounds under study were determined using Mass spectrometer (Model: POLARIS Q; Serial no: MS 211912) coupled with trace GC ultra gas chromatograph (Thermo Fisher Scientific India Pvt. Ltd., Model: Trace GC Ultra 320080111$).$ A DB-5MS column $(30 \mathrm{~m} \times 0.25 \mathrm{~mm}$ ID $\times 0.25 \mu \mathrm{m}$ film thicknesses with stationary phase $5 \%$ Phenyl polysilphenylene siloxane was used. Helium gas of $99.999 \%$ purity was used as a carrier gas at a flow rate of $1 \mathrm{ml} / \mathrm{min}$ with a linear velocity of $10 \mathrm{ml} / \mathrm{s} .1 \mu \mathrm{l}$ extracted sample was injected with autosampler (Model no: AI3000) into the column in a split mode. Initially the temperature of $\mathrm{GC}$ oven was programmed at $50^{\circ} \mathrm{C}$ with a hold time of $1 \mathrm{~min}$ and gradually raised to $300^{\circ} \mathrm{C}$ at the rate of rising temperature $80^{\circ} \mathrm{C} / \mathrm{min}$ with a hold time of $5 \mathrm{~min}$ and was finally raised to $320^{\circ} \mathrm{C}$ at the rate of rising temperature $10^{\circ} \mathrm{C} / \mathrm{min}$ with a hold time of $10 \mathrm{~min}$. The MS (mass spectra) was carried out in the electron impact mode (EI) at $70 \mathrm{eV}$. Keeping the temperature at $250^{\circ} \mathrm{C}$, the detector was set at $40-600 \mathrm{D}$. Mass spectrum of GC-MS was interpreted using the database of National Institute Standard and Technology (NIST) harbouring $1,50,000$ patterns. With the aid of database information and the data store software XCALIBUR, the name of the compound of the experimental materials, molecular weight and structure were determined.

\section{Validation of the method}

Standard solutions of all four selected compounds were run before analysis of each sample to assess the linearity of the profiling method. A standard solution containing a mixture of JA, IAA, SA and ABA (Sigma-Aldrich, USA) was prepared. The calibration graph was obtained in the concentration range of the standard phytohormones (Table. 1).

For reproducibility, all samples were run on the same day because sample preparation procedure and instrumental analysis can contribute to the variability of the method adopted.

For recovery, the chemical stability of the metabolites/derivatives and the exraction method were assessed. In this recovery test, double amounts of standard compounds were mixed in the leaf-extract at the initial stage of the extraction procedure. Both tissue mixes and unspiked extracts from the same test samples were then run to compare and calculate the recovery rates. The eluted compounds were characterized on the basis of their molecular formula, structure, retention time, and percent relative peak area. Processed data were subjected to statistical analysis (the term significant is used only when $\mathrm{p}<0.05$ according to the t-test embedded in Microsoft Office Excel).

\section{RESULTS AND DISCUSSION}

GC-MS based metabolite study is still considered the most versatile platform with many advantages over other analytical methods in respect to high separation power and reproducibility [12,13]. The method adopted here to detect the most abundant phytohormones in leaf sample of tobacco and tomato. Several studies have shown that between different phytohormones, there are synergistic as well as antagonistic actions in plants [24].

Enough reports are available authenticating that in the regulation process of plant growth and development, there are signaling crosstalks between several plant hormones besides their individual actions.

\section{Hormonal changes in response to salt stress}

Based on GC-MS results (Figures 3 and 4), salt stress has been found to have elevated levels of IAA, ABA, SA and JA in leaves of tobacco and tomato compared to the control. Endogenous level of all signaling molecules under study was found higher in tomato than in tobacco.

ABA is a well documented phytohormone for stress responses. Its signaling pathway is the core of salt stress response in plant system. ABA-mediated signaling has a vital role to play in plant responses to different adverse environmental conditions (abiotic constraints) and also biotic stresses like plant pathogens [25]. producing more ABA than tomato under stress (Figures 3-5).

Table 1: Fragmented parameters and obtained values in GC-MS method for quantification of phytohormones

\begin{tabular}{|c|c|c|c|c|c|c|c|c|}
\hline \multirow[t]{2}{*}{ Analyte } & \multicolumn{2}{|c|}{$\begin{array}{l}\text { Standard concentration }(\mathrm{ng} / \mathrm{ml}) \\
(n=3)\end{array}$} & \multicolumn{2}{|c|}{$\begin{array}{c}\text { Tobacco }(n g / g ~ F W) \\
(n=3)\end{array}$} & \multicolumn{2}{|c|}{$\begin{array}{c}\text { T omato }(\mathrm{ng} / \mathrm{g} \mathrm{FW}) \\
(n=3)\end{array}$} & \multirow[t]{2}{*}{ Retention time (min) } & \multirow[t]{2}{*}{ ion $\mathrm{m} / \mathrm{z}$} \\
\hline & Range of conc & Linearity & Control & Treated & Control & Treated & & \\
\hline IAA & $1-50$ & 0.9887 & 3 & 2.1 & 15.2 & 18 & 2.04 & 141 \\
\hline SA & $1-50$ & 0.9878 & 8.8 & 25.5 & 101 & 138 & 5.45 & 120 \\
\hline$A B A$ & $5-100$ & 0.9954 & 80 & 150 & 421 & 440.4 & 12.06 & 200 \\
\hline JA & $5-100$ & 0.9988 & 3 & 5.7 & 25 & 28 & 22.71 & 183 \\
\hline
\end{tabular}




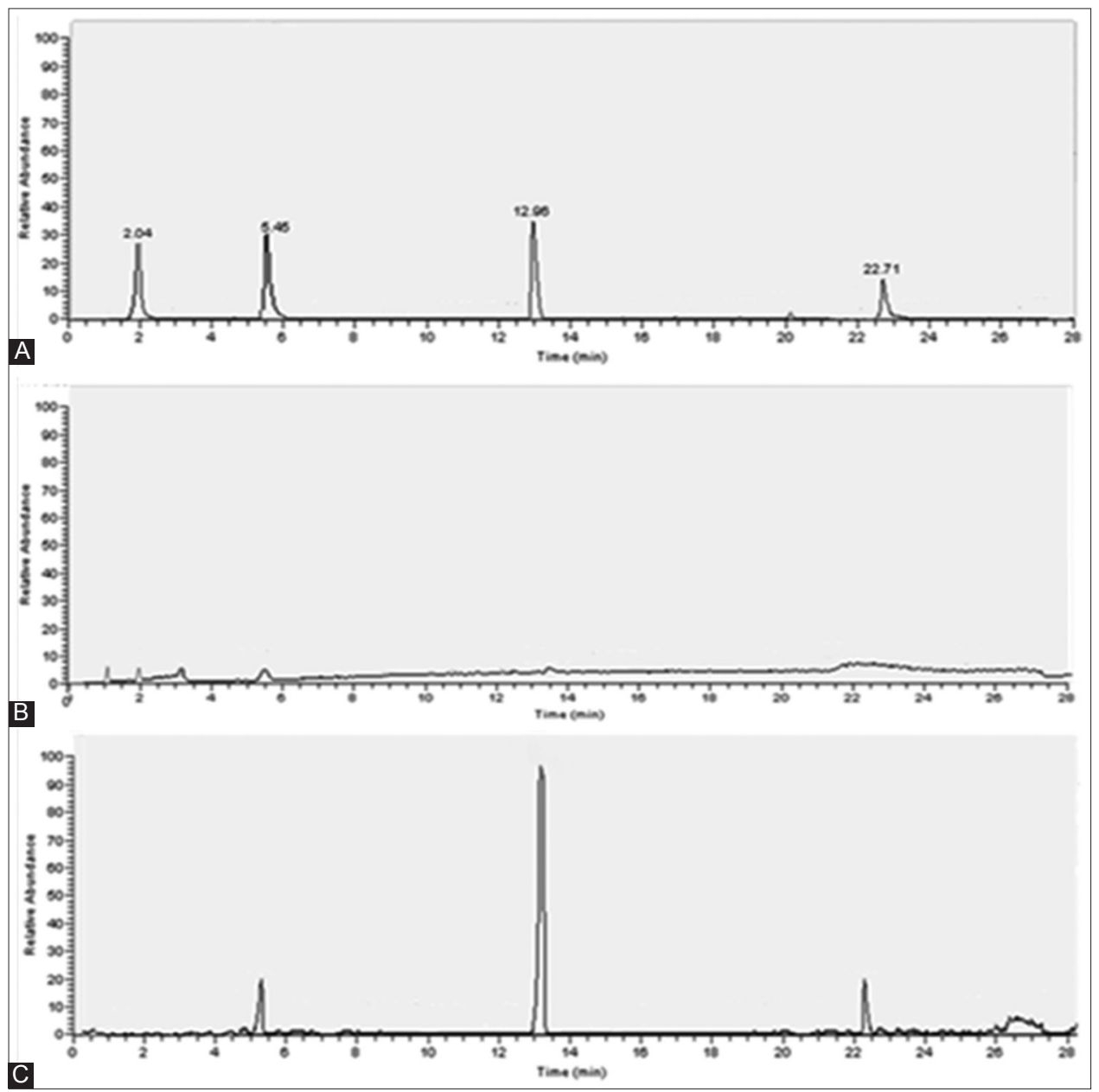

Figure 3: Representative GC chromatograms of selected plant hormones. This study with leaf sample shows that tobacco is detection and quantification of plant hormones by using GC-MS. A) Standards of phytohormones showing the peaks of IAA (indole acetic acid), ABA (abscisic acid), SA (salicylic acid) and JA (jasmonic acid); B) ethyl acetate extract of untreated leaf sample of tobacco; C) ethyl acetate extract of $150 \mathrm{mM}$ $\mathrm{NaCl}$ treated leaf sample of tobacco displaying the chromatographic peaks in the range as compared with the standard.

The result may be because tomato is moderately salt tolerant. Previous study [26] with wheat also showed lesser enhancement of ABA under stress. So in different plant species ABA synthesis and catabolism are regulated differently. ABA is also produced in the roots where the plant may be under stress. ABA is then translocated from roots to the aerial parts/leaves and there it rapidly alters the osmotic potential of the guard cells of the stomata, resulting into stomatal shrinkage and closure. The rate of transpiration is reduced by ABA-induced stomatal closure. This phenomenon prevents further water loss from the leaves at the time of water scarcity [27]. As also described by He and Cramer [28], salt withstanding plants produce low amount of ABA than sensitive ones and can perform normally during moderate salinity [29].

IAA level of occurrence in our findings is of quite variable in nature (Figure 5). IAA is known to be involved in response to salinity in crop plants. According to the GC-MS result (Figures 3 and 4) $\mathrm{NaCl}$ treatments resulted in a marginal increase of IAA in tomato but decreased in tobacco. The difference between two plant species can be perceived by noting the ability of the plants in resisting the rise of IAA level in leaves under salinity [30], and a higher level of IAA also has been correlated with retarded growth [31]. A study shows $75 \%$ reduction in IAA level in tomato while under salinity stress [32]. A report suggests significant reduction of this regulator in crop plants such as rice and tomato [33]. Another report shows that salinity caused reduction in IAA levels in maize plants but SA application could enhance them effectively [34]. According to an another study, elevation of IAA (auxin) level by overexpression of auxin biosynthetic related YUCCA3 caused hypersensivity to salt stress [35]. Several investigations have shown that auxins have the ability to increase stress tolerance in various plant species.

$\mathrm{SA}$ is considered as the stress hormone and also has been recognised as a contributor to improved plant abiotic stress tolerance [36]. The result demonstrates (Figure 5) that SA increased 3-fold in tobacco whereas tomato shows 1.5-fold enhancement. So under stress, elevation is lesser in tomato in comparison to tobacco leaves. SA has been identified for its involvement in plat response to abiotic stress (e.g. drought, salinity cold, and heat) [37,38]. Previous study [39] has shown that several proteins were induced by SA in cucumber. The expression of these proteins were found to be involved in cell defence, carbohydrate metabolism, photosynthesis, 


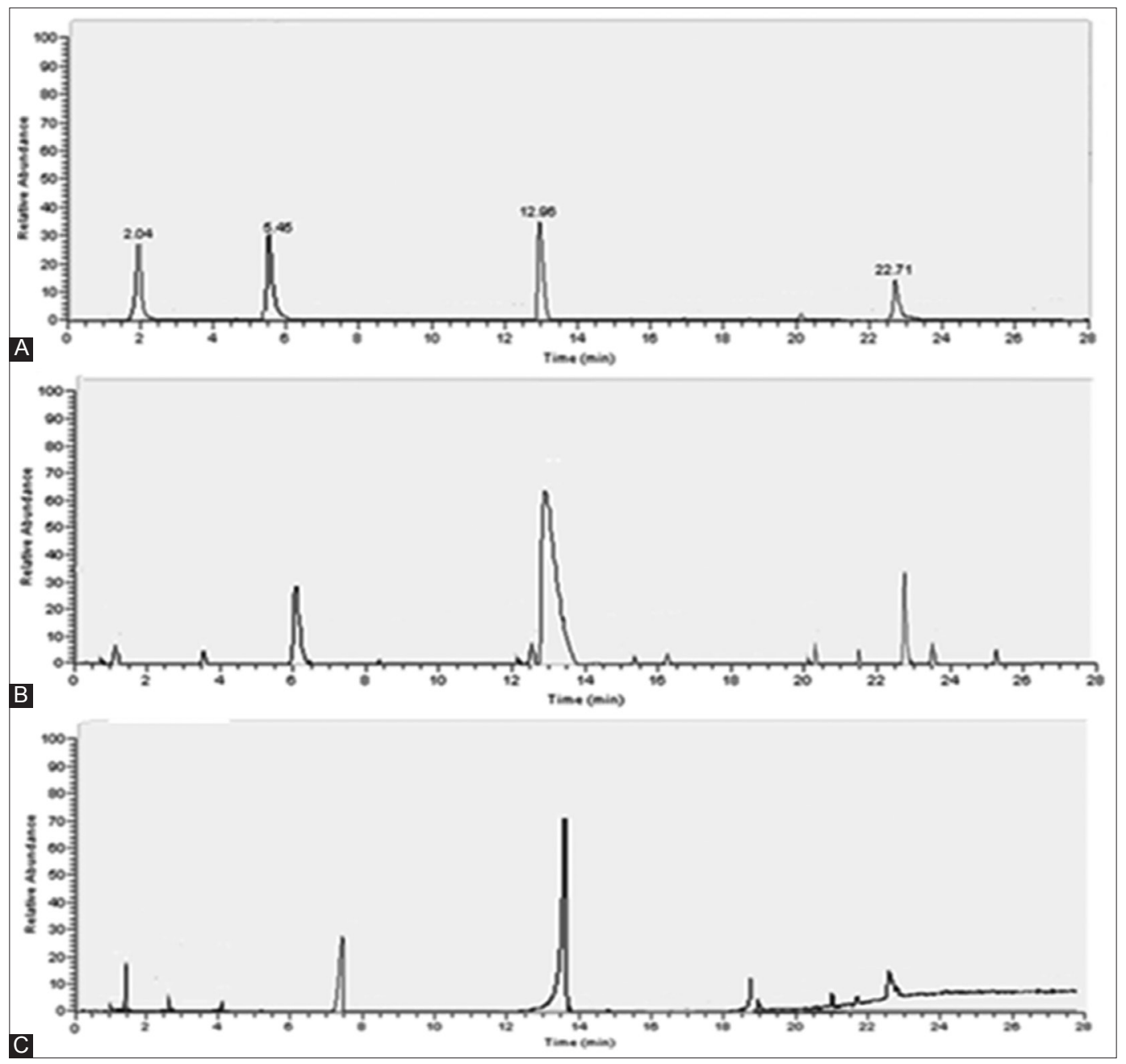

Figure 4: Representative GC chromatograms of selected plant hormones. Detection and quantification of plant hormones by using GC-MS. A) Standards of phytohormones showing the peaks of IAA (indole acetic acid), ABA (abscisic acid), SA(salicylic acid) and JA (jasmonic acid); B) ethyl acetate extract of untreated leaf sample of tomato; C) ethyl acetate extract of $150 \mathrm{mM} \mathrm{NaCl}$ treated leaf sample of tobacco displaying the chromatographic peaks in the range as compared with the standard.

antioxidative reactions, respiration and energy homeostasis, protein folding and bigenesis. Alteration of SA in two species under study indicates that tomato here is being less affected by the stress imposed. Endogenous level of SA increased significantly in tobacco. SA in general, involved in the process of defence mechanism [40]. A report suggests that endogenous SA increased salt stress tolerance in wheat seedlings [41]. SA induction could ameliorate abiotic stress such as water deficit on cell membrane by upregulating ABA and proline [42].

JAs have major function in the abiotic and biotic stresses as well as $[43,44]$. This plant growth regulation includes senescence, reduced growth, tendril coiling, flower development and leaf abscission. Based on our GC-MS analysis (Figures 1 and 2), it was noted that JA level was significantly increased, reaching to more than 1.5-fold in salt treated tobacco compared to the control (Figures 3 and 5). Here in treated tomato leaves, no significant elevation of JA was detected (Figures 4 and 5). The results indicate that abiotic stress like salinity differentially modulates the endogenous level of JA. There are sporadic reports of evaluation that plant's responses under stress in this regard [45].
Comparatively lesser is known about JA's role in abiotic stresses in relation to biotic stress. According to a study done, JA signaling is also active in the response to different abiotic stresses [46]. JA signaling research performed in wheat plant suggests that JA has an important role in getting rid of potentially harmful ROSs [47-49]. Zhao et al. [50] demonstrated salinity tolerance in wheat via the JA signaling pathway. Other studies demonstrated that jasmonate levels were enhanced under stresses [51,52]. A study on rice brought out that, both low availability of water and high salinity stresses resulted in an induced and enhanced jasmonate levels in the leaves and roots $[53,54]$. Another research in rice reported overexpression of OsJAZ6 improved salt and mannitol stresses [55]. Ismail et al. [45] suggested that salt stress response may be modulated by jasmonate. The present study is in accordance with several other experiments cites that JA can differentially regulate the plants' responses and adaptation to various kinds of abiotic stresses.

\section{Cross-talk of IAA, SA, ABA and JA}

The plant hormones such as auxins, cytokinins (CKs), gibberellins (GAs), abscisic acid (ABA), salicylic acid (SA), jasmonic acid (JA), 


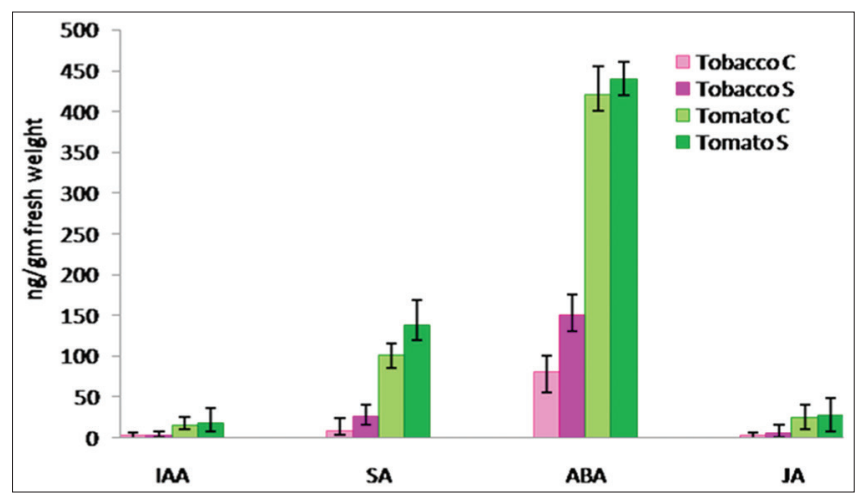

Figure 5: Salt stress alters the hormonal balance of leaves. Comparison of the phytohormones in control(C) and stressed(S) leaf tissue. The abundance of the endogenous phytohormones IAA, ABA, JA and SA. Determination of the level of phytohormones present in the control and salt-stressed tissue of Tobacco and Tomato, demonstrates that GC-MS method is highly helpful in obtaining the dynamic range of phytohormones with small amounts of starting material $(200 \mathrm{mg}$ ). Values are means \pm SD ( $n=3$ independent samples.

ethylene (ET), and brassinosteroids (BRs) respond to diverse stresses through synergistic and antagonistic ways. This interaction between various phytohormones often referred to as signaling cross talk. In fact, the response mechanism to different stresses not solely restricted to these hormones only. Recent researches provide clues that the interactions of these hormones with each other and with other hormones are increasingly more complex than previously thought in various crop species. In plants, abiotic stress response regulated by phytohormone-signaling depends upon several factors like stress type, duration of exposure and intensity. ABA is known to be occupying the key position as a hormone regulator in stresses. The cross-talk between SA and JA was initially observed in tomato responding to wound [56]. SA, JA take active roles in plant abiotic stress tolerance, whereas IAAs are involved in stress tolerance of both kinds [57, 58, 38]. SA and JA are biochemically linked signaling molecules. Their induction can be triggered by stresses and become integral part of plant defence responses [59]. JA, SA also interact with $\mathrm{ABA}$ to trigger stomatal closure and thereby restricting water loss during osmotic stresses $[60,61,62]$. In an experiment on Arabidopsis, Miao and Zentgraf [63] demonstrated that two pathways of SA and JA interact during senescence and regulate a senescence-responsive TF, WRKY53 antagonistically. A previous research found that inspite of being antagonistic, both SA and JA signaling pathways simultaneously become active in certain conditions [64]. Our findings propose that in the process of stress responses, phytohormone signaling is critical for homeostasis and for maintaining a fine balance between ABA, IAA, SA and JA. In this experiment tomato seems more efficient in keeping hormonal balance in the shoot parts. On the contrary tobacco responds with higher alteration in the hormonal accumulation in leaves against salinity stress.

\section{CONCLUSIONS}

With the great advantage of sensitivity and simplicity of the powerful tool GC-MS, the individual hormones IAA, ABA, JA or SA targeted here for quantitative measurement in two different plant species. Differences in the accumulation of chemical signals (IAA, ABA, JA and SA) in tobacco and tomato, is possibly due to the differences in their ability to stress resistance. Though in minor amount but these molecules have significant roles in balancing plants behaviour under salinity stress. The same method should be applicable to a broad range of other regulating factors or metabolites of interest. The versatility of the technique is important. Moreover, analysis of altered levels of signaling molecules at different experimental conditions in our study is providing more precise insight into the dynamics of regulatory processes. Present study may be helpful in pronouncing the fate of selected phytohormones. It also may assist in evaluating the capacity of detoxification in various plant species. The underlying molecular mechanism of signaling networks between ABA and other hormones is complex and more comprehensive study is needed in future. In this direction, phytohormone engineering seems promising for plant biologists.

\section{ACKNOWLEDGMENTS}

Authors thank Division of Plant Biology and the Director of the Bose Institute. The authors acknowledge the support of the Central Instrument Facility of the Bose Institute.

\section{REFERENCES}

1. Rushton PJ, Bokowiec MT, Han S, Zhang H, Brannock JF, Chen X, Laudeman TW, Timko MP. Tobacco transcription factors: novel insights into transcriptional regulation in the Solanaceae. Plant Physiol 2008;147:280-295.

2. Carvalho PCdeF, Dewulf A K MY, Moraes A de, Bremm C, Trindade JKda, Lang C R. Kikuyo grass potential to maintain milk yield and quality from cows receiving decreasing levels of supplements. Rev Bras Zootec 2010;39(9):1866-1874.

3. Salhi A, Negrão S, Essack M, Morton Mitchell JL, Bougouffa S, Razali R, Radovanovic A, Marchand B, Kulmanov M, Hoehndorf R, Tester M, Bajic VB. DES-TOMATO: A Knowledge Exploration System Focused On Tomato Species. Scientific reports 2017;7 (1):5968.

4. Arc E, Sechet J, Corbineau F, Rajjou L, Marion-Poll A. ABA crosstalk with ethylene and nitric oxide in seed dormancy and germination. Frontiers in Plant Science 2013b;4:63.

5. McAtee P, Karim S, Schaffer R, David K. A dynamic interplay between phytohormones is required for fruit development, maturation, and ripening. Frontiers in Plant Science 2013;4:79.

6. Denancé N, Sánchez-Vallet A, Goffner D, Molina A. Disease resistance or growth: the role of plant hormones in balancing immune responses and fitness costs. Front Plant Sci 2013

7. Raghavendra AS, Gonugunta VK, Christmann A, Grill E. ABA perception and signalling. Trends Plant Sci 2010;15:395-401.

8. Franklin K A. Shade avoidance. New Phytol 2008;179:930-944.

9. Skirycz A, Inzé D. More from less: plant growth under limited water. Curr. Opin. Biotechnol 2010;21:197-203.

10. Wani SH, Kumar V, Shriram V, Sah SK. Phytohormones and their metabolic engineering for abiotic stress tolerance in crop plants. Crop J 2016;4:162-176.

11. Wilkinson S, Davies WJ. ABA-based chemical signalling: the coordination of responses to stress in plants. Plant Cell Environ 2002;25:195-210.

12. Tiwari S, Prasad V, Chauhan PS, Lata C. Bacillus amyloliquefaciens confers tolerance to various abiotic stresses and modulates plant response to phytohormones through osmoprotection and gene expression regulation in rice. Front Plant Sci 2017; 8:1510.

13. Wolters H, Jurgens G. Survival of the flexible: hormonal growth control and adaptation in plant development. Nature Reviews Genetics 2009;10:305-317.

14. Gazzarrini S, McCourt P. Genetic interactions between ABA, ethylene and sugar signaling pathways. Curr Opin. Plant Biol 2001;4:387-391.

15. Saniewski M, Czapski J. Stimulatory effect of methyl jasmonate on 
ethylene production. Experientia 1985;41:256-257.

16. Mussig C, Biesgen C, Lisso J, Uwer U, Weiler EW, Altmann T. A novel stress-inducible 12-oxo-phytodienoate reductase from Arabidopsis thaliana provides a potential link between brassinosteroid-action and jasmonic-acid synthesis. J Plant Physiol 2000;157:143-152.

17. Yang SF, Hoffman NE. Ethylene biosynthesis and its regulation in higher plants. Annu. Rev. Plant Physiol. 1984;35:155-189.

18. Zarembinski TI, Theologis A. Ethylene biosynthesis and action: a case of conservation. Plant Mol. Biol 1994; 26:1579-1597.

19. Cary AJ, Liu W, Howell SH. Cytokinin action is coupled to ethylene in its effects on the inhibition of root and hypocotyls elongation in Arabidopsis thaliana seedlings. Plant Physiol 1995;107:1075-1082.

20. Ross J, O'Neill D. New interactions between classical plant hormones. Trends Plant Sci 2001: 6:2-3.

21. Müller A, Düchting P, Weiler EW. A multiplex GC-MS/MS technique for the sensitive and quantitative single-run analysis of acidic phytohormones and related compounds, and its application to Arabidopsis thaliana. Planta 2002; 216:44-56.

22. Lin L, Luo S, Luan T, Wang X, Zou S. Determination of phytohormones in plant extracts using in-matrix ethyl chloroformate derivatization and DLLME-GC-MS. LCGC Eur 2013; 26:310-324.

23. Murashige T, Skoog F. A revised medium for rapid growth and bioassays with tobacco tissue cultures. Physiol Plant 1962; 15:473-497

24. Moubayidin L, Di Mambro R, Sabatini S. Cytokinin-auxin crosstalk. Trends Plant Sci 2009; 14:557-562.

25. Seo M, Koshiba T. Complex regulation of ABA biosynthesis in plants. Trends in Plant Science 2002; 7(1): 41-48.

26. Xuemei JI, Dong B. B Shiran, MJ Talbot, JE Edlington, T Hughes, RG White, F Gubler, R Dolferus. Control of ABA catabolism and ABA homeostasis is important for reproductive stage stress tolerance in cereals. Plant physiology 2011; 156: 647-662.

27. Munemasa S, Hauser F, Park J, Waadt R, Brandt B, Schroeder JI. Mechanisms of abscisic acid-mediated control of stomatal aperture. Curr Opin Plant Biol 2015; 28: 154-162.

28. He T, Cramer GR. Abscisic acid concentrations are correlated with leaf area reduction in two salt-stressed rapid-cycling Brassica species. Plant Soil 1996; 179:25-33.

29. Kefu Z, Munns R, King RW. Abscisic acid levels in NaCl-treated barley cotton and saltbush. Aust J Plant Physiol 1991; 18:17-24.

30. Ribaut JM, Pilet PE. Effects of water stress on growth, osmotic potential and abscisic acid content of maize roots. Physiol Plant $1991 ; 81: 156-162$

31. Ribaut JM, Pilet PE. Water stress and indole-3ylacetic acid content of maize roots. Planta 1994; 193:502-507

32. Dunlap JR, Binzel ML. NaCl reduces indole-3-acetic acid levels in the roots of tomato plants independent of stress-induced abscisic acid. Plant Physiol 1996; 112:379-384.

33. Kazan K. Auxin and the integration of environmental signals into plant root development. Ann Bot 2013; 112:1655-1665.

34. S Fahad, A Bano, Effect of salicylic acid on physiological and biochemical characterization of maize grown in saline area. Pak J Bot 2012; 44:1433-1438.

35. Jung J, Park C. Auxin modulation of salt stress signaling in Arabidopsis seed germination. Plant Signal Behav 2011; 6:1198-1200.

36. Khan MIR, Fatma M, Per TS, Anjum NA, Khan NA. Salicylic acidinduced a biotic stress tolerance and underlying mechanisms in plants. Front Plant Sci 2015; 6:462.

37. M Fujita, Y Fujita, Y Noutoshi, F Takahashi, Y Narusaka, K YamaguchiShinozaki, K Shinozaki. Crosstalk between abiotic and biotic stress responses: a current view from the points of convergence in the stress signaling networks. Curr Opin Plant Biol 2006; 9:436-442.

38. Miura K, Tada Y. Regulation of water, salinity, and cold stress responses by salicylic acid. Front Plant Sci 2014; 5:4.

39. Hao JH, Donga CJ, Zhanga ZG, Wanga XL, Shang QM. Insights into salicylic acid responses in cucumber (Cucumis sativus L.) cotyledons based on a comparative proteomic analysis. Plant Sci 2011; 187: 69-82.

40. Wasternack C, Hause B. Jasmonates: biosynthesis, perception, signal transduction and action in plant stress response, growth and development. Ann Bot 2013; 111(6):1021-1058.

41. Shakirova FM, Sakhabutdinova AR, Bezrukova MV, Fatkhutdinova RA, Fatkhutdinova DR. Changes in the hormonal status of wheat seedlings induced by salicylic acid and salinity. Plant Sci 2003;
164:317-322.

42. Bandurska $H$, Stroinski $A$. The effect of salicylic acid on barley response to water deficit. Acta Physiol Plant 2005; 27:379-386.

43. Turner JG, Ellis C, Devot A. The jasmonate signal pathway. Plant Cell 2002; S153-S164.

44. Pauwels L, Inze D and Goossens A. Jasmonate-inducible gene: what does it mean? Trends Plant Sci 2009; 14:87-91.

45. Ismail A, Riemann M, Nick P. The jasmonate pathway mediates salt tolerance in grapevines. Journal of Experimental Botany 2012; 63:2127-2139.

46. Ahmad P, Rasool S, Gul A, Sheikh SA, Akram NA, Ashraf M, Kazi AM, Gucel S. Jasmonates: multifunctional roles in stress tolerance. Front. Plant Sci 2016; 7:813.

47. Kan, GZ, Li GZ, Liu GQ, Xu W, Peng XQ, Wang CY. Exogenous salicylic acid enhances wheat drought tolerance by influence on the expression of genes related to ascorbate-glutathione cycle. Biol Plant 2013; 57:718-724

48. Qiu Z, Guo J, Zhu A, Zhang L, Zhang M. Exogenous jasmonic acid can enhance tolerance of wheat seedlings to salt stress. Ecotoxicol Environ Saf 2014; 104:202-208.

49. Shan $C$, Zhou $Y$, Liu M. Nitric oxide participates in the regulation of the ascorbate-glutathione cycle by exogenous jasmonic acid in the leaves of wheat seedlings under drought stress. Protoplasma 2015; 252:1397-1405

50. Zhao $Y$, Dong $W$, Zhang N, Ai X, Wang M, Huang Z. A wheat allene oxide cyclase gene enhances salinity tolerance via jasmonate signaling. Plant Physiol 2014; 164:1068-1076.

51. Creelman RA, Mullet JE. Jasmonic acid distribution and action in plants: Regulation during development and response to biotic and abiotic stress. Proc Natl Acad Sci USA 1995; 92:4114-4119.

52. Wang Y, Mopper S, Hasentein KH. Effects of salinity on endogenous ABA, IAA, JA, and SA in Iris hexagona. J Chem Ecol 2001; 27:327-342.

53. Moons A, Prinsen E, Bauw G, Montagu MV. Antagonistic effects of abscisic acid and jasmonates on salt stress-inducible transcripts in rice roots. Plant Cell 1997; 9:2243-2259.

54. Tani T, Sobajima H, Okada K, Chujo T, Arimura S, Tsutsumi N, Nishimura M, Seto H, Nojiri H, Yamane H. Identification of the OsOPR7 gene encoding 12-oxophytodienoate reductase involved in the biosynthesis of jasmonic acid in rice. Planta 2008; 227:517-526.

55. Ye H, Du H, Tang N, Li X, Xiong L. Identification and expression profiling analysis of TIFY family genes involved in stress and phytohormone responses in rice. Plant Mol Biol 2009a; 71: 291-305.

56. Harms K, Ramirez I, Pena-Cortés H. Inhibition of wound-induced accumulation of allene oxide synthase transcripts in flax leaves by aspirin and salicylic acid. Plant Physiology 1998; 118:1057-1065.

57. Du H, Liu H, Xiong L. Endogenous auxin and jasmonic acid levels are differentially modulated by abiotic stresses in rice. Front Plant Sci 2013a; 4:397.

58. Joshi R, Singla-Pareek SL and Pareek A. Engineering abiotic stress response in plants for biomass production. J Biol Chem 2018; 293(14):5035-5043.

59. Khan MIR, Syeed S, Nazar R, Anjum NA. An insight into the role of Salicylic Acid and Jasmonic Acid in Salt Stress Tolerance. In Phytohormones and Abiotic Stress Tolerance in Plants (pp. 277-300). Berlin. Springer Publishing; 2012.

60. Hossain MA, Munemasa S, Uraji M, Nakamura Y, Mori IC, Murata Y. Involvement of endogenous abscisic acid in methyl jasmonate-induced stomatal closure in Arabidopsis. Plant Physiol 2011; 156:430-438.

61. Miura K, Okamoto H, Okuma E, Shiba H, Kamada H, Hasegawa PM, Murata Y. SIZ1 deficiency causes reduced stomatal aperture and enhanced drought tolerance via controlling salicylic acid-induced accumulation of reactive oxygen species in Arabidopsis. Plant Journal 2012; 73:91-104.

62. Qi T, Wang J, Huang H, Liu B, Gao H, Liu Y, Song S, Xie D. Regulation of jasmonate-induced leaf senescence by antagonism between bHLH subgroup IIle and IIId factors in Arabidopsis. Plant Cell 2015; 27:1634-1649

63. Miao Y, Zentgraf $U$. The antagonist function of Arabidopsis WRKY53 and ESR/ESP in leaf senescence is modulated by the jasmonic and salicylic acid equilibrium. The Plant Cell 2007; 19: 819-830.

64. Tsuda K. Division of Tasks: Defense by the Spatial Separation of Antagonistic Hormone Activities, Plant and Cell Physiology 2018; 59(1): 3-4. 